\title{
Non-HLA antigens and HLA-DR matching in corneal transplantation
}

The continuing uncertainty about the benefits of HLA matching in corneal transplantation will be familiar to corneal surgeons. This has persisted despite numerous clinical studies conducted over the past 20 years to define the best matching strategies. Some of these studies have shown no advantage in matching, even in high risk cases. Others have found matching at A and B locus antigens beneficial, while the outcome of DR matching has been very inconsistent. These apparently conflicting findings are no doubt partly due to differences in study design, such as the criteria for patient selection, treatment regimens, or whether studies were prospective or retrospective. However, even the most recent, carefully designed trials using sophisticated statistical analysis have brought us no nearer to a definitive answer. For example, the US Collaborative Corneal Transplantation Study, ${ }^{1}$ involving high risk cases, high doses of topical immunosuppressants, and very careful patient follow up, found no benefit in HLA matching. A Danish HLA-DR matching study that was retrospective, but where typing was by the precise molecular (RFLP) method, ${ }^{2}$ rather than by serology, indicated that DR matching was beneficial in high risk cases. In contrast, prospective $\mathrm{UK}^{3}$ and French ${ }^{4}$ studies (involving serological typing) have shown a detrimental effect of DR matching. These findings contrast with those in renal transplantation, where the HLA matching effect is still apparent, even with the improvements in immunosuppression afforded by cyclosporin-DR compatibility being paramount. ${ }^{56}$

One might well wonder why, for a relatively privileged tissue, in which recourse to systemic immunosuppression is generally unnecessary, HLA matching has not solved, or at least greatly diminished, the remaining problems of rejection. It is not merely that the high average survival rate of corneal transplants masks the beneficial effect of matching in difficult cases, because the value of matching is uncertain even in the categories at highest risk of rejection. This is particularly unfortunate, because the prognosis for such patients is as poor as for high risk recipients of other organs and, at least in some centres in the UK, is increasingly considered too poor to merit transplantation. ${ }^{7}$

Most of us are aware that every transplanted tissue or organ is unique in ways that influence not only the likelihood but also the mechanism of rejection. Such features may well define the mismatches that are most likely to lead to rejection. However, this idea has not been applied to decisions about matching strategies in corneal transplantation or, indeed, to the design of matching studies. Rather, procedures developed for other organs have been followed. Perhaps we should now pause to consider more carefully the special characteristics of the cornea that might alter the outcome of HLA matching in comparison with other organs.

A series of animal experiments reported several years ago gave some indication that matching might not yield the expected benefits. Katami et $a l^{8}$ studied rejection, without immunosuppression, in a variety of rat strains of different major histocompatibility (MHC) type (that is, differing in the rat equivalent of HLA). Graft survival when donor and recipient pairs were of one particular MHC disparity was
11 days. Survival in a strain combination with the same MHC disparity as previously, but with different 'background' genetic differences, was more than 100 days. This indicated that genes other than those of the MHC (that is, non-MHC genes, sometimes called minor genes) play an important role in graft rejection. Further studies using strains matched at all MHC loci showed that non-MHC mismatches alone led to rejection of half (48\%) of grafts. There was still a benefit of MHC matching, because full mismatching resulted in rejection of $85 \%$ of grafts. Independently of Katami et al, Nicholls et al, using a backcross model and a stronger genetic disparity, found that 95\% of MHC matched grafts were ultimately rejected, although rejection was significantly delayed compared with fully mismatched grafts, all of which were rejected. ${ }^{9}$ Use of this model enabled us to show that at least three independently segregating non-MHC genes were involved. We also found that immunosuppression prolonged survival of MHC mismatched grafts more readily than those that were matched, thus diminishing, though not abrogating, the value of matching. ${ }^{10}$

More recent experimental work using mice has confirmed a prominent role for non-MHC antigens in corneal graft rejection. ${ }^{11}$ The antigens themselves remain largely uncharacterised, although the chromosomal locations of some 50 or more have been determined in mice by monitoring skin graft rejection between strains differing at a single genetic locus. The best known non-MHC antigens in humans are blood group antigens, but these are atypical in that they are targeted by soluble antibody, rather than by $T$ lymphocytes. Their role in corneal graft rejection is equivocal. ${ }^{12}$ Non-MHC antigens targeted by $T$ cells are known in humans, being responsible for rejection of HLA identical bone marrow and kidney grafts and graft versus host disease. They are thought to be intracellular proteins that are polymorphic-that is, slightly different in different individuals, and which are digested and displayed on the cell surface in the form of small peptides in the grooves of MHC class I or II molecules. A partial genetic analysis of some of these antigens has been performed in humans by Schreuder and coworkers, using $\mathrm{T}$ cells lines derived from bone marrow recipients. ${ }^{13}$ Some cell lines were specific for the male antigen $(\mathrm{H}-\mathrm{Y})$. This has recently been identified as the product of a gene on the long arm of the $\mathrm{Y}$ chromosome, which differs from the homologous protein from the $\mathrm{X}$ chromosome by some 200 amino acids. ${ }^{14}$ The amino acid sequence of the protein suggests that it may function as a gene transcription factor. Goulmy et al also found that at least four non-MHC loci are associated with graft versus host disease, ${ }^{15}$ and preliminary tests on corneas showed that two non-HLA specific $T$ cell lines, one of which recognised $\mathrm{H}-\mathrm{Y}$, were able to kill $\mathrm{MHC}$ matched corneal cells. ${ }^{16}$ However, the identity and role of non-MHC antigens in corneal transplantation remain to be fully elucidated. As well as being targets for rejection, such genes may also act by moderating the immune response to other antigens.

So why should so called 'minor' antigens play a disproportionate role in corneal graft rejection and why should 
the results of class II matching be so equivocal? The answers may lie, paradoxically, in the relative lack of $\mathrm{MHC}$ class II (HLA-D in humans) bearing dendritic cells in the donor cornea, one of the factors know to contribute to its immunological privilege. It was well established in the 1980 s that the most potent stimulus to rejection of a renal graft were the donor MHC class II bearing dendritic cells in the graft. ${ }^{17}$ These are so called 'professional' antigen presenting cells, which initiate an immune response by presenting antigen to $\mathrm{CD}^{+}$(helper) $\mathrm{T}$ cells. In an allograft, such cells can present their own alloantigen, either in the form of mismatched MHC or mismatched bound peptide, directly to recipient $T$ cells. There is no need for the alloantigen to be shed from the graft and picked up, processed, and presented by recipient antigen presenting cells, which would be analogous to the way that pathogen antigens are presented. Since the main alloantigen in this direct form of presentation would be a mismatched MHC class II molecule, it is not surprising that matching for class II reduces rejection of organs, such as the kidney, that are rich in these class II bearing cells.

In corneas, however, which contain few class II bearing dendritic cells, this direct pathway is of diminished importance. It is therefore predictable that class II matching would not confer the same benefit as it would in renal transplantation. On the other hand, the periphery of the recipient cornea and, in an inflamed and vascularised cornea, the graft bed itself contain numerous dendritic cells, ${ }^{18}$ 19 which are well placed to pick up and present antigen by the indirect route. ${ }^{18}$ Moreover, the presence of these cells is associated with corneal graft failure and rejection. ${ }^{18}{ }^{20}$ In the epithelium these are the familiar Langerhans cells, also present in skin. Similar cells are found in the stroma. In rats, such cells rapidly infiltrate the donor cornea itself after transplantation and are augmented during rejection (Figueiredo et al, unpublished data). Such cells in the recipient bed would have relatively easy access to graft antigens, which are likely to be released in substantial amounts by the trauma of the operation, the healing process, and by removal of sutures. Once loaded with antigen, dendritic cells would migrate to the draining lymph node via the conjunctival lymphatics or, in an inflamed bed, via lymphatic vessels invading the cornea and there initiate an immune response. In a vascularised organ graft, recipient antigen presenting cells, entering the graft from the general circulation, only very slowly replace those of the donor, so that donor antigen presenting cells would predominate for a long period of time after transplantation. Thus, the relative importance of recipient and donor antigen presenting cells is reversed in corneas compared with vascularised organs; in corneas those of the recipient playing the dominant role.

Class II matching may be less beneficial for corneas than for vascularised organs, but can we envisage circumstances where it might actually be detrimental? The answer is 'yes', given that recipient cells play the dominant antigen presenting role. This is because presentation of antigen (expressed as peptide in the class II binding groove) by recipient cells, if they are class II matched with the graft, generates $\mathrm{CD}^{+} \mathrm{T}$ cells of the correct specificity to bind to those same antigens expressed on a class II expressing graft cell. The greatest chance of rejection would obviously be where there are numerous other mismatches - that is, both class I and multiple non-MHC (and in humans, mismatching at the other untyped loci, HLA-C, HLA-DR, and HLA-DQ). The potential for MHC class I to be presented in this indirect way, in the binding groove of a matched class II molecule, offers an explanation as to why HLA-A and HLA-B matching has, in general, seemed beneficial, while DR matching has not-that is, class II matching pro- motes rejection in this situation, while class I matching reduces it. Moreover, only if class II is matched does the indirect pathway generate $\mathrm{T}$ cells of the correct specificity to recognise non-MHC antigens on the graft. Thus, matching for class II theoretically makes rejection on account of non-MHC mismatches more likely and we have an explanation as to why such antigens may play a relatively important role in corneal graft rejection. The extent to which non-MHC antigens play a role in a particular donor-recipient combination will depend on the number of non-MHC mismatches and whether class II is matched. Conversely, the extent to which class II matching prejudices graft survival will depend on the extent of other disparities between donor and recipient.

Obviously, graft destruction mediated directly by $\mathrm{CD}^{+}$ $\mathrm{T}$ cells is only facilitated if class II is actually expressed on the graft endothelium during rejection. There have been several reports that class II expression is induced in humans under conditions of inflammation, including graft rejection. ${ }^{21}$ In the LEW to PVG rat strain combination, which we have used, such expression appears stronger than class I expression, which is not only relatively weak, but seems to be only mildly upregulated, if at all, during rejection (Figueiredo et al, unpublished data). Graft destruction by $\mathrm{CD}^{+} \mathrm{T}$ cells could occur via a delayed type hypersensitivity response-that is, the cells adhering to the graft and producing cytokines to promote the non-specific cytotoxic action of macrophages. Alternatively, the $\mathrm{CD} 4^{+}$cells themselves could be directly cytotoxic, without involvement of other cells. Unfortunately, there is still little direct evidence as to the cell types responsible for killing corneal cells. Histological examination of grafts undergoing rejection reveals a mixed cell infiltrate, including $\mathrm{CD}^{+}$(cytotoxic) $\mathrm{T}$ cells and it is likely that more than one killing mechanism operates. Moreover, the relative contribution of each cell type will vary according to the particular mismatches involved. However, the central role of $\mathrm{CD}^{+} \mathrm{T}$ cells has been confirmed indirectly by Ayliffe et $a{ }^{22}$ who found that depletion of these cells in rats prevented rejection, while depletion of $\mathrm{CD}^{+}$cells did not.

It is certainly true that progress in understanding corneal graft rejection has been painfully slow. While there is little doubt that in the cornea there is bias in favour of recipient antigen presentation, we cannot totally exclude a role for donor antigen presenting cells. More human and animal studies are needed to investigate this and fully resolve the matching dilemma. The present clinical choices are either to use heavy immunosuppression in high risk cases, as in the CCTS study, ${ }^{1}$ perhaps obviating the need for matching, or to develop a matching strategy that works. In theory, this might ultimately involve matching for HLA-A and HLA-B while deliberately mismatching for HLA-DR or even typing and matching for selected nonMHC antigens. The latter is currently not possible and, in any case, would seem prohibitively expensive. Unlike the MHC, which is extremely polymorphic, non-MHC loci generally have only two alleles, one of which is a null allele (that is, the antigen is lacking). Thus, graft rejection would only result from such a mismatch if the recipient possessed the null allele. However, although the polymorphism at each locus is much less than for HLA, typing for such antigens is more difficult than for HLA or blood group antigens, as specific $T$ cell lines, rather than antibodies, are required. However, it is possible that one or two immunodominant antigens may be responsible for the non-MHC effect in humans, in which case such typing might ultimately be feasible in very high risk cases.

Meanwhile, based on the best available evidence at present, derived from the UK Corneal Transplant Follow up Study, the UKTSSA Corneal Advisory Group recom- 
mends that matching for class I (HLA-A and HLA-B) should be offered in high risk cases. Limitation of matching to only the A and B loci will maximise the donor pool available and minimise waiting times. Recommendations about class II (HLA-DR) matching must await the outcome of further studies.

Department of Ophthalmology,

School of Medical Sciences

University Walk, Bristol BS8 1TD

1 The Collaborative Corneal Transplantation Studies Research Group. Effectiveness of histocompatibility matching in high-risk corneal transplantation. Arch Ophthalmol 1992;110:1392-403.

2 Baggesen K, Ehlers N, Lamm LU. HLA-DR/RFLP compatible corneal Baggesen K, Ehlers N, Lamm LU. HL

3 Vail A, Gore SM, Bradley BA, Easty DL, Rogers CA, Armitage WJ, on behalf of the Corneal Transplant Follow-up Study Collaborators. Influence of donor and histocompatibility factors on corneal graft outcome. Transplantation 1995;58:1210-7.

4 Assouline M, Delbosc B, Rigal D, Adenis JP, Bechetoille A, Sabatier P, et al. Survival of high risk HLA matched penetrating keratoplasty. Vision Res 1995;85 (suppl):S83.

5 Opelz G. Influence of HLA matching on survival of second kidney transplants in cyclosporin-treated recipients. Transplantation 1989;47:823-7.

6 Dyer PA, Johnson RWG, Martin S, Bakran A, Gokal R, Harris R, et al. Evidence that matching for HLA antigens significantly increases transplant survival in 1001 renal transplants performed in the northwest region of survival in 1001 renal transplants perfo

7 Larkin DFP. Corneal allograft rejection. Brf Ophthalmol 1994;78:649-52.

8 Katami M, White DJG, Watson PG. An analysis of corneal graft rejection in the rat. Transplant Proc 1989;21:3147-9.

9 Nicholls SM, Bradley BA, Easty DL. Effect of mismatches for major histocompatibility complex and minor antigens on corneal graft rejection. Invest Ophthalmol Vis Sci 1991;32:2729-34.
10 Nicholls SM, Bradley BA, Easty DL. Apparent resistance to immunosuppression of MHC-matched corneal transplants. Transplantation 1995;59. 325-8.

11 Sonoda Y, Streilein JW. Orthotopic corneal transplantation in miceevidence that the immunogenetic rules of rejection do not apply. Transplantation 1992;54:694-704.

12 Batchelor JR, Casey TA, Werb A, Gibbs D, Prasad SS, Lloyd, et al. HLA matching and corneal grafting. Lancet 1976;i:551-4.

13 Schreuder GMT, Pool J, Blokland E, van Els C, Bakker A, van Rood J, et al. A genetic analysis of human minor histocompatibility antigens demonstrates Mendelian segregation independent of HLA. Immunogenetics 1993; 38:98-105.

14 Wang W, Meadows LR, den Haan JMM, Sherman NE, Chen Y, Blokland E, et al. Human H-Y: a male-specific histocompatibility antigen derived from the SMCY protein. Science 1995;269:1588-90.

15 Goulmy E, Schipper R, Pool J, Blokland E, Falkenburg JH, Vossen J, et al. Mismatches of minor histocompatibility antigens between HLA-identical donors and recipients and the development of graft-versus-host disease after bone marrow transplantation. $N$ Engl F Med 1996;334:281-5.

16 Goulmy E, Pool J, van Lochem E, Volker-Dieben H. The role of human minor histocompatibility antigens in graft failure: a mini-review. Eye 1995; 9:180-4.

17 Lechler RI, Batchelor JR. Restoration of immunogenicity to passenger celldepleted kidney allografts by the addition of donor strain dendritic cells. $\mathcal{F}$ Exp Med 1982;155:31-41.

18 Willams KA, Mann TS, Ash JK, Coster DJ. The role of resident accessory cells in corneal allograft rejection in the rabbit. Transplantation 1986;42: 667-71.

19 Williams KA, Ash JK, Coster DJ. Histocompatibility antigen and passenger cell content of normal and diseased human cornea. Transplantation 1985;39:265-9.

20 Williams, KA, White MA, Ash JK, Coster DJ. Leucocytes in the graft bed are associated with corneal graft failure: analysis by immunohistology and actuarial graft survival. Ophthalmology 1989;96:38-44.

21 Pepose JS, Gardiner, Nestor MS, Foos RY, Pettit TH. Detection of HLA class I and II antigens in rejected human corneal allografts. Ophthalmology 1985;92:1480-4.

22 Ayliffe W, Alam Y, Bell EB, McLeod D, Hutchinson IV. Prolongation of rat corneal graft survival by treatment with anti-CD4 monoclonal antibody. $B r$ f Ophthalmol 1992;76:602-6.

\section{The ophthalmic physician: an indulgence or a necessity?}

Surgical advances in ophthalmology have been spectacular during the past few decades and have radically changed the practice of ophthalmology. Accordingly, the late 20th century trainee devotes much of his time rightly honing his skills in surgical manoeuvres and most of the subspecialisation in ophthalmology is surgically orientated, as emphasised by the standards and knowledge expected in fellowship examinations and for higher surgical training in ophthalmology. However, all ophthalmologists are aware that a large part of our practice relates to medical problems for which there is no surgical treatment. Many fields of general medicine are involved such as rheumatology, neurology, endocrinology and diabetology, cardiovascular disease, and inflammatory/infectious disease. Some of the conditions which are included in these spheres of practice, such as keratoconjunctivitis sicca and infectious anterior segment disease, can be adequately treated by the 'primary care ophthalmologist' with appropriate subspecialist referral when indicated. However, as a result of recent research developments, treatments and cures for many medical ophthalmic conditions are now available and it therefore becomes a major responsibility to ensure that these treatments are properly delivered and monitored. Patient management and research development into these conditions have, to date, been performed with liaison between ophthalmologist and physicians or by a few ophthalmologists who have chosen to take that path. Ophthalmologists have to feel comfortable knowing that they can adequately manage and treat patients without overburdening our equally stretched physician colleagues.

Currently the major blinding disease in the developed world is macular degeneration. However, in the working age population diabetic retinopathy and chronic uveitis represent two major causes of blindness. While most ophthalmologists feel confident in their management of the former, the fact that the latter is a curable/preventable cause of blindness is less well recognised. Treatment of chronic uveitis is now best achieved with long term immunosuppression but few ophthalmologists have the experience to feel secure in the long term use of drugs such as cyclosporin A and methotrexate, while physicians are not trained to recognise the ophthalmological end points which can determine necessary changes to treatment regimens.

A new kind of ophthalmologist is therefore required: one who is skilled in ophthalmic medical diagnosis, in relating systemic medical disease to ophthalmic conditions, in assessing the general medical status of patients who have primary ophthalmological disease, and in prescribing the most appropriate drug therapies and monitoring schedules for treatment of medical sight threatening diseases.

Recognition of the need for medical ophthalmologists has resulted in the Royal College of Ophthalmologists and Royal College of Physicians jointly establishing a professional training programme leading to accreditation and registration on the specialist register in the new subspecialty of medical ophthalmology - that is, a training for the ophthalmic physician. Currently, entry to the new training programme may be gained from a background in general medicine or general ophthalmology and the training programme appropriately tailored in line with a general template which ensures full exposure to the various aspects of medical ophthalmology. This training programme is in its infancy, but it is already clear that the route of entry to a medical ophthalmic training programme is less important than how we are going to provide training. Relevant postgraduate general medical training at some stage, however, appears appropriate. When medical ophthalmology as a specialty has become sufficiently well established in the number of trained specialists, it will be in a better posi- 